\title{
Crystal structure of 2-amino-3-nitro-pyridinium dihydrogenmonophosphate, $\mathrm{C}_{5} \mathrm{H}_{8} \mathrm{~N}_{3} \mathrm{O}_{6} \mathrm{P}$
}

\section{S. Akriche and M. Rzaigui*}

Faculté des Sciences de Bizerte, Laboratoire de Chimie des Matériaux, 7021 Zarzouna Bizerte, Tunisia

Received May 12, 2000, CCDC-No. $1267 / 460$
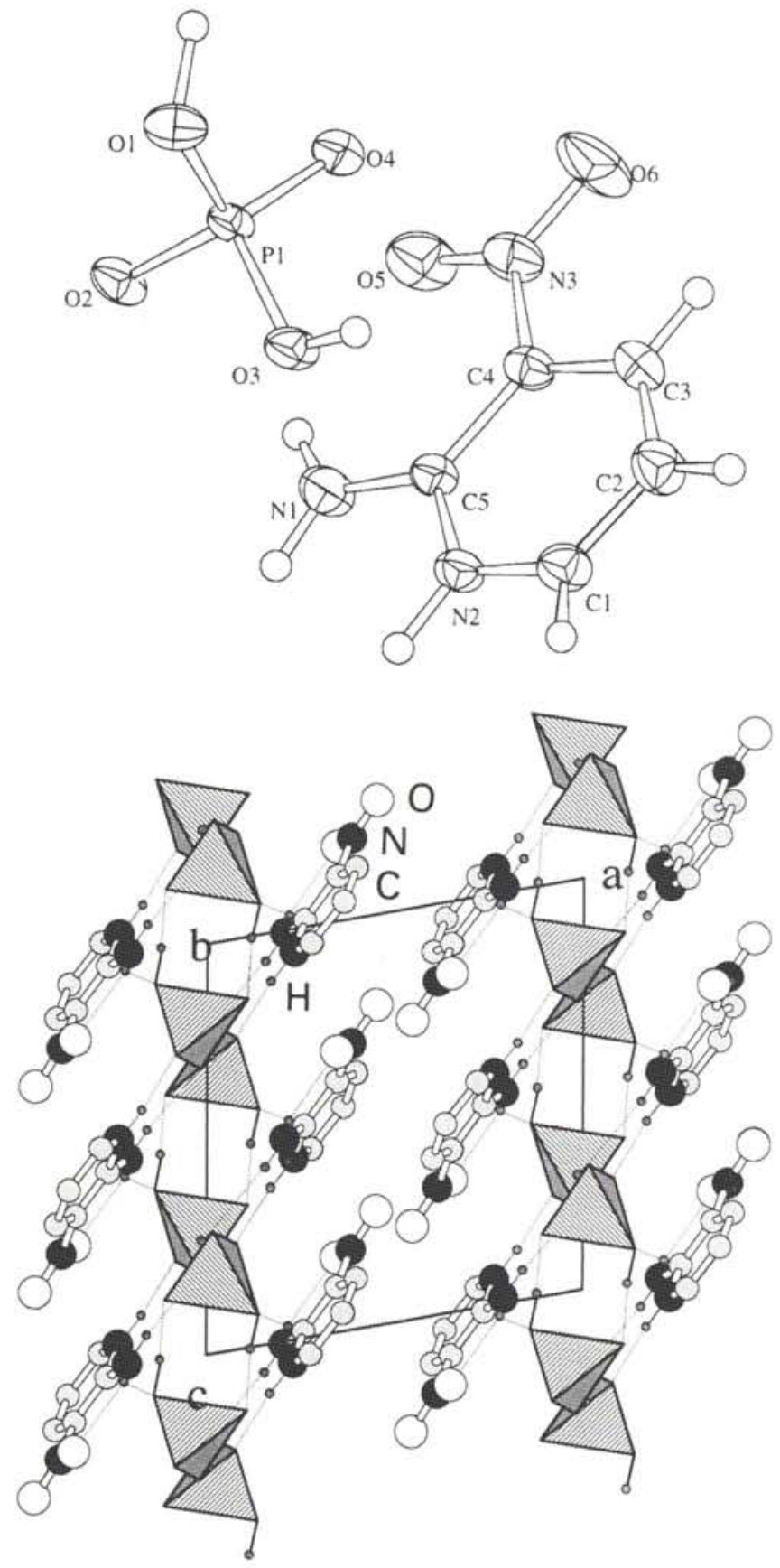

\begin{abstract}
$\mathrm{C}_{5} \mathrm{H}_{8} \mathrm{~N}_{3} \mathrm{O}_{6} \mathrm{P}$, monoclinic, $P 12_{1} / c 1$ (No. 14), $a=9.710(3) \AA$, $b=9.254(1) \AA, c=10.373(6) \AA, \beta=100.36(5)^{\circ}, V=916.9 \AA^{3}$, $Z=4, R_{\mathrm{gt}}(F)=0.040, w R_{\mathrm{ref}}(F)=0.057, T=296 \mathrm{~K}$.
\end{abstract}

\section{Source of material}

The 2-amino-3-nitropyridine was dissolved in $20 \mathrm{~cm}^{3}$ of an acidic solution containing an excess of $\mathrm{H}_{3} \mathrm{PO}_{4}(\mathrm{~d}=1.5)$ heated to $373 \mathrm{~K}$. The so obtained solution is immediately concentrated by evaporation in air-circulation for some hours then maintained for many days at room temperature till formation of prismatic yellow crystals.

\section{Discussion}

The structure of $\left(\mathrm{C}_{5} \mathrm{H}_{6} \mathrm{~N}_{3} \mathrm{O}_{2}\right) \mathrm{H}_{2} \mathrm{PO}_{4}$ can be described as a stacking of thick layer centred by $(b, c)$ plane. Inside one layer, the $\mathrm{H}_{2} \mathrm{PO}_{4}{ }^{-}$anions, connected by strong hydrogen bonds, are assembled to build infinite layers perpendicular to the $a$ direction. The organic groups $\left(\mathrm{C}_{5} \mathrm{H}_{6} \mathrm{~N}_{3} \mathrm{O}_{2}\right)^{+}$, are anchored onto two phosphoric layers through multiple hydrogen bonds to make up into two dimensions infinite network.

Table 1. Data collection and handling.

\section{Crystal:}

Wavelength:

$\mu$ :

Diffractometer, scan mode:

$2 \theta_{\max }$ :

$N(h k l)_{\text {measured, }} N(h k l)_{\text {unique: }}$ Criterion for $I_{\text {obs }}, N(h k l)$ gt:

$N(\text { param })_{\text {refined: }}$

Programs:

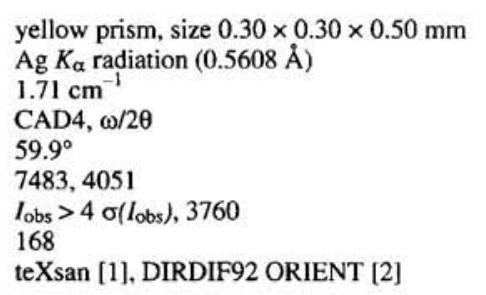

Table 2. Atomic coordinates and displacement parameters (in $\AA^{2}$ ).

\begin{tabular}{lllrll}
\hline Atom & Site & $x$ & \multicolumn{1}{l}{$y$} & \multicolumn{1}{l}{$z$} & \multicolumn{1}{l}{$U_{\text {iso }}$} \\
\hline $\mathrm{H}(1)$ & $4 e$ & $0.755(1)$ & $0.308(1)$ & $0.903(1)$ & $0.042(3)$ \\
$\mathrm{H}(2)$ & $4 e$ & $0.604(1)$ & $0.367(1)$ & $1.048(1)$ & $0.064(4)$ \\
$\mathrm{H}(3)$ & $4 e$ & $0.547(1)$ & $0.180(1)$ & $1.184(1)$ & $0.038(3)$ \\
$\mathrm{H}(4)$ & $4 e$ & $0.841(1)$ & $-0.138(1)$ & $0.925(1)$ & $0.075(4)$ \\
$\mathrm{H}(5)$ & $4 e$ & $0.786(1)$ & $-0.207(1)$ & $1.034(1)$ & $0.043(3)$ \\
$\mathrm{H}(6)$ & $4 e$ & $0.824(1)$ & $0.095(1)$ & $0.874(1)$ & $0.062(4)$ \\
$\mathrm{H}(7)$ & $4 e$ & $0.985(1)$ & $0.218(1)$ & $1.221(1)$ & $0.058(3)$ \\
$\mathrm{H}(8)$ & $4 e$ & $1.110(2)$ & $0.046(2)$ & $1.513(2)$ & $0.084(5)$ \\
& & & & & \\
\hline
\end{tabular}

* Correspondence author (e-mail: Mohamed.Rzaigui@fsb.rnu.tn) 
Table 3. Atomic coordinates and displacement parameters (in $\AA^{2}$ ).

\begin{tabular}{|c|c|c|c|c|c|c|c|c|c|c|}
\hline Atom & Site & $x$ & $y$ & $z$ & $U_{11}$ & $U_{22}$ & $U_{33}$ & $U_{12}$ & $U_{13}$ & $U_{23}$ \\
\hline $\mathbf{P}(1)$ & $4 e$ & $1.02892(2)$ & $0.02214(2)$ & $1.30687(2)$ & $0.03621(7)$ & $0.01849(6)$ & $0.02186(6)$ & $-0.00007(5)$ & $0.01204(5)$ & $-0.00063(5)$ \\
\hline$O(1)$ & $4 e$ & $1.13906(6)$ & $0.07549(6)$ & $1.42371(5)$ & $0.0457(3)$ & $0.0362(3)$ & $0.0287(2)$ & $-0.0126(2)$ & $0.0084(2)$ & $-0.0019(2)$ \\
\hline$O(2)$ & $4 e$ & $1.08088(6)$ & $-0.10801(5)$ & $1.24177(5)$ & $0.0521(3)$ & $0.0190(2)$ & $0.0344(2)$ & $0.0009(2)$ & $0.0223(2)$ & $-0.0021(2)$ \\
\hline$O(3)$ & $4 e$ & $1.01591(7)$ & $0.14413(6)$ & $1.20022(5)$ & $0.0591(3)$ & $0.0224(2)$ & $0.0284(2)$ & $0.0077(2)$ & $0.0189(2)$ & $0.0047(2)$ \\
\hline$O(4)$ & $4 e$ & $0.88969(6)$ & $-0.00257(6)$ & $1.34866(5)$ & $0.0347(2)$ & $0.0408(3)$ & $0.0297(2)$ & $0.0003(2)$ & $0.0115(2)$ & $0.0033(2)$ \\
\hline$O(5)$ & $4 e$ & $0.65210(8)$ & $-0.20782(7)$ & $1.17964(7)$ & $0.0721(4)$ & $0.0359(3)$ & $0.0527(3)$ & $-0.0067(3)$ & $0.0272(3)$ & $0.0080(3)$ \\
\hline$O(6)$ & $4 e$ & $0.54782(9)$ & $-0.04831(9)$ & $1.27655(8)$ & $0.0840(4)$ & $0.0641(5)$ & $0.0740(4)$ & $-0.0029(4)$ & $0.0607(3)$ & $0.0095(4)$ \\
\hline$N(1)$ & $4 e$ & $0.79138(8)$ & $-0.12989(8)$ & $0.99031(7)$ & $0.0575(4)$ & $0.0330(3)$ & $0.0398(3)$ & $0.0103(3)$ & $0.0240(3)$ & $0.0064(2)$ \\
\hline$N(2)$ & $4 e$ & $0.76704(7)$ & $0.11084(7)$ & $0.94129(6)$ & $0.0354(3)$ & $0.0315(3)$ & $0.0274(2)$ & $0.0003(2)$ & $0.0122(2)$ & $0.0034(2)$ \\
\hline$N(3)$ & $4 e$ & $0.61529(7)$ & $-0.08389(8)$ & $1.19336(7)$ & $0.0399(3)$ & $0.0435(3)$ & $0.0362(3)$ & $-0.0100(3)$ & $0.0168(2)$ & $0.0033(2)$ \\
\hline $\mathrm{C}(1)$ & $4 e$ & $0.72031(8)$ & $0.24532(8)$ & $0.95468(7)$ & $0.0432(4)$ & $0.0292(3)$ & $0.0338(3)$ & $-0.0022(3)$ & $0.0116(3)$ & $0.0024(2)$ \\
\hline $\mathrm{C}(2)$ & $4 e$ & $0.63535(9)$ & $0.27694(9)$ & $1.04263(8)$ & $0.0467(4)$ & $0.0314(3)$ & $0.0422(4)$ & $0.0033(3)$ & $0.0151(3)$ & $-0.0023(3)$ \\
\hline $\mathrm{C}(3)$ & $4 e$ & $0.60150(8)$ & $0.16488(9)$ & $1.12001(7)$ & $0.0341(3)$ & $0.0390(3)$ & $0.0343(3)$ & $-0.0003(3)$ & $0.0136(2)$ & $-0.0049(3)$ \\
\hline$C(4)$ & $4 e$ & $0.65261(7)$ & $0.02786(8)$ & $1.10793(6)$ & $0.0291(3)$ & $0.0331(3)$ & $0.0266(2)$ & $-0.0046(2)$ & $0.0094(2)$ & $0.0002(2)$ \\
\hline$C(5)$ & $4 e$ & $0.73858(7)$ & $-0.00378(7)$ & $1.01410(6)$ & $0.0305(3)$ & $0.0305(3)$ & $0.0253(2)$ & $-0.0009(2)$ & $0.0086(2)$ & $0.0015(2)$ \\
\hline
\end{tabular}

\section{References}

1. Molecular Structure Corporation. teXsan for Windows version 1.03. Single Crystal Structure Analysis Software. Version 1.03. MSC, 3200 Research Forest Drive, The Woodlands, TX 77381, USA 1997.

2. Beurskens, P. T.; Admiraal, G.; Beurskens, G.; Bosman, W. P.; Garcia-Granda, S.; Gould, R. O.; Smits, J. M. M.; Smykalla, C.: The DIRDIF program system, Technical Report of the Crystallography Laboratory, University of Nijmegen, The Netherlands 1992. 